\title{
The Utilization of Core Exercises in Patients With Patellofemoral Pain: A Critically Appraised Topic
}

\author{
Emma F. Zuk, Gyujin Kim, Jacqueline Rodriguez, Brandon Hallaway, Amanda Kuczo, Shayna Deluca, \\ Kirsten Allen, Neal R. Glaviano, and Lindsay J. DiStefano
}

\begin{abstract}
Clinical Scenario: Patellofemoral pain (PFP) is characterized by general anterior knee pain around the patella and is one of the most prevalent knee conditions. PFP is challenging to treat due to a wide range of contributing factors and often has chronic, reoccurring symptoms. Traditional treatment focuses on quadriceps and gluteal strengthening with minimal emphasis on deep trunk musculature. Recently, there has been a growing body of literature supporting the beneficial effects of core stability exercises as a treatment option for PFP. Clinical Question: Are core stability exercises coupled with traditional rehabilitation more effective than only traditional rehabilitation techniques for decreasing pain in patients with PFP? Summary of Key Findings: Three articles met the inclusion criteria and investigated core strengthening exercises as a treatment for PFP. Two studies investigated a 4-week exercise protocol and demonstrated a greater decrease in pain when compared to the control group. The third study examined the effects of a 6-week program where both the intervention and control groups resulted in similar reduction of pain. All articles included received a minimum of 6 on the PEDro scale. Clinical Bottom Line: There is evidence that supports core stability exercise protocols coupled with traditional rehabilitation as being more effective in reducing pain in patients with PFP when compared to traditional rehabilitation alone. Strength of Recommendation: The grade of A is recommended based on the Strength of Recommendation Taxonomy.
\end{abstract}

\section{Clinical Scenario}

Patellofemoral pain (PFP) is the common term for general knee pain relating to the front of the knee and around the patella. ${ }^{1} \mathrm{PFP}$ is one of the most commonly diagnosed knee conditions, with this phenomenon most often appearing in young, physically active individuals with females being at an increased risk. ${ }^{1-4}$ PFP is a challenging condition to treat due to multiple contributing factors related to the patient's functional impairments and potentially causal factors, such as strength, range of motion, or neuromuscular control. ${ }^{1}$ While the etiology is unknown, PFP is theorized to be due to increased stress at the patellofemoral joint.

Traditional rehabilitation programs have focused on the knee and hips in hopes of improving neuromuscular control around the knee. ${ }^{5}$ Patients will often complete exercises aimed at strengthening their gluteal and quadriceps muscles. ${ }^{6}$ These interventions demonstrate success in the short term, with improvements in both pain and subjective function. However, $70 \%$ to $90 \%$ of patients diagnosed with PFP will have reoccurring symptoms demonstrating the need for more effective long-standing treatment interventions. ${ }^{1}$ This highlights that PFP is a multifactorial problem, where no single strategy is appropriate for every patient. Therefore, advancements to current treatment strategies are warranted to improve the outcomes for these patients.

Current treatment interventions include targeting the proximal muscles of the hip due to their role in both hip and knee control. However, these interventions often omit the other muscles of the lumbopelvic-hip complex that have various attachments to the pelvis. Targeting multiple muscles of the core could improve pelvic stability and neuromuscular control down the kinetic chain,

The authors are with the University of Connecticut, Storrs, CT, USA. Zuk (emma. zuk@uconn.edu) is corresponding author. minimizing stress on the patellofemoral joint. Core stability exercises typically focus on strengthening and activating the abdominal muscles, as well as the surrounding muscles, including the spine and hip musculature. There is a growing body of evidence to suggest that improving core strength and stability will result in greater reductions in pain for patients with PFP..$^{7-9}$

\section{Focused Clinical Question}

Are core stability exercises, coupled with traditional rehabilitation, more effective in reducing pain in patients with PFP compared with traditional rehabilitation protocols alone?

\section{Summary of Search, "Best Evidence" Appraised, and Key Findings}

- The literature search was conducted to only include "highquality" evidence (PEDro $\geq 6$ ) that investigated rehabilitation protocols which included core stability in patients with PFP (https://pedro.org.au/wp-content/uploads/PEDro_ scale.pdf).

- A total of 5 studies were found during the initial search, with 3 studies being included in the final analyses. All 3 included studies were single-blinded randomized control trials where the therapist was blinded to the patients allocated group.

- Of the 3 included studies, 2 studies earned a PEDro score of $6^{7,8}$ and one earned a $7 .{ }^{9}$

- Ferber et al ${ }^{7}$ implemented a $6-\mathrm{d} / \mathrm{wk}$ exercise protocol for 6 weeks comparing a knee-focused rehabilitation group and a hip/corefocused rehabilitation group. The knee-focused rehabilitation included non-weight-bearing to weight-bearing quadriceps strengthening exercises. The hip/core rehabilitation included 
non-weight bearing, muscle-strengthening exercises that focused on activating hip musculature and progressed to participants stabilizing their core muscles prior to conducting weight-bearing, core-strengthening, and balance exercises. Both the hip/core and knee groups improved PFP pain using the visual analog scale (VAS; 3.11 and 2.98, respectively) and Anterior Knee Pain Scale (12.58 and 12.90, respectively) that exceeded the minimal clinically important change thresholds for both outcome measures. However, the hip/core group demonstrated a significant improvement 1 week prior when compared with the knee-focused group.

- Motealleh et $\mathrm{al}^{9}$ employed a 3 exercise sessions per day protocol for 4 weeks comparing a core-focused rehabilitation with a traditional rehabilitation program. The traditional rehabilitation program focused on strengthening the quadriceps and hamstring muscles. In addition to the traditional rehabilitation program, the core group performed exercises focused on neuromuscular training of the deep abdominal muscles responsible for activating the core. There were greater improvements in pain for the core rehabilitation program and the traditional rehabilitation program based on the VAS (4 and 3, respectively) and Anterior Knee Pain Scale (18.5 and 12, respectively).

- Chevidikunnan et $\mathrm{al}^{8}$ implemented a 3-d/wk intervention for 4 weeks for a core group and a traditional rehabilitation group. The core intervention consisted of curl-ups, side bridges, and quadrupedal stance (bird dog exercises). The traditional rehabilitation intervention consisted of eccentric quadriceps strengthening and abductor and external rotators strengthening. There were greater improvements in pain in the core rehabilitation group when compared with the traditional rehabilitation group based on the VAS (4.2 and 5.1, respectively).

\section{Clinical Bottom Line}

There is strong evidence supporting that core stability exercise protocols coupled with traditional rehabilitation are effective in improving pain in patients with PFP. It is important to note that all subjects, regardless of group, demonstrated a decrease in pain. Based on the current evidence, interventions that include core stability exercises over a minimum of 4 weeks decrease pain in patients with PFP faster than traditional rehabilitation alone. Therefore, clinicians should consider incorporating various core exercises when developing therapeutic exercise interventions for patients with PFP.

\section{Strength of Recommendation}

There is "grade A" evidence that rehabilitation programs that incorporate core exercises provide significantly greater improvements in pain for those experiencing PFP than traditional rehabilitation protocols in accordance with the Strength of Recommendation Taxonomy (https://www.aafp.org/dam/AAFP/ documents/journals/afp/SORTGuidance.pdf).

\section{Search Strategy}

\section{Terms Used to Guide Search Strategy}

- Patient/client group: men or women diagnosed with PFP

- Intervention: core stability exercises coupled with traditional rehabilitation

- Comparison: traditional PFP management/rehabilitation

- Outcomes: pain

\section{Sources of Evidence Searched}

- PubMed

- Literature cross-referenced for further resources

\section{Inclusion and Exclusion Criteria}

\section{Inclusion Criteria}

- Studies with participants diagnosed with PFP

- Participants must be between 16 and 45 years of age

- Studies with pain as an outcome measure

- Studies with core stability exercises in the intervention

- PEDro score $\geq 6$

- Articles published after 2015

- Literature written in English

\section{Exclusion Criteria}

- Studies with unclear PFP diagnosis

- Participants out of the age range

- Studies with patients with previous diagnosed knee injury history

- Studies without core stability exercises within the intervention

\section{Results of Search}

A total of 5 relevant studies were located and categorized as shown in Table $1 .^{6-10}$ However, after further inspection, 3 studies utilized

Table 1 Summary of Study Designs of Articles Retrieved

\begin{tabular}{lcl}
\hline Level of evidence & Study design/methodology of articles retrieved & Study \\
\hline $1 \mathrm{~b}$ & $\mathrm{RCT}$ & ${\text { Ferber et } \mathrm{al}^{7}}^{7}$ \\
$\mathrm{lb}$ & $\mathrm{RCT}$ & ${\text { Earl-Boehm }(2018)^{\mathrm{a}}}^{\mathrm{a}}$ \\
$\mathrm{lb}$ & $\mathrm{RCT}$ & ${\text { Bolgla }(2016)^{\mathrm{a}}}^{9}$ \\
$\mathrm{lb}$ & $\mathrm{RCT}$ & Motealleh et al $^{9}$ \\
$\mathrm{lb}$ & $\mathrm{RCT}$ & Chevidikunnan et al $^{8}$ \\
\hline
\end{tabular}

Abbreviation: RCT, randomized control trial.

${ }^{\text {a }}$ Study met initial inclusion criteria but was removed due to identical data from original study by Ferber et al. 
Table 2 Characteristics of Included Studies

\begin{tabular}{|c|c|c|}
\hline & Ferber et al $^{7}$ & Motealleh et al $^{9}$ \\
\hline Design & RCT & RCT \\
\hline Participants & $\begin{array}{l}\text { A total of } 199 \text { participants }(66 \text { males and } \\
133 \text { females; age }=29[7] \mathrm{y}, \text { height }=170.4 \\
{[9.4] \mathrm{cm} \text {, weight }=67.6[13.5] \mathrm{kg} \text { ) with PFP }} \\
\text { consistent with inclusion and exclusion } \\
\text { criteria used for PFP-related research were } \\
\text { used in this study. }\end{array}$ & $\begin{array}{l}\text { A total of } 28 \text { participants, all female, with } \\
\text { unilateral PFP diagnosed by an orthopedic } \\
\text { specialist (intervention group: age }=28 \\
\text { [6] y, height }=161[6] \mathrm{cm} \text {, weight }=58.5 \\
\text { [8.8] kg; traditional rehabilitation group: } \\
\text { age }=30[6] \mathrm{y} \text {, height }=159 \text { [5] cm, weight } \\
=58.7[7.9] \mathrm{kg} \text { ) were used in this study. }\end{array}$ \\
\hline
\end{tabular}

Intervention Participants were randomly assigned to a investigated 6-wk hip- and core-focused $(\mathrm{n}=111)$ or knee-focused $(\mathrm{n}=88)$ rehabilitation group. Exercises were to be completed in a minimum of $6 \mathrm{~d} / \mathrm{wk}$. Compliance was monitored. Participants were allowed to visit the athletic trainer 3 times/wk. PFP patients in the hip and core group performed nonweight-bearing, muscle-strengthening exercises that focused on activating hip musculature. These progressed to patients being instructed to stabilize their core musculature before initiation in weightbearing exercises including core-strengthening and balance exercises to activate core musculature. Participants in the knee group performed non-weight-bearing quadriceps strengthening that progressed to weightbearing quadriceps-strengthening exercises.

Outcome VAS, Anterior Knee Pain Scale, and measures muscle strength and core endurance Level of $1 b$

evidence

Validity

score

Main findings
Participants were divided into 2 groups: intervention group (core neuromuscular training in addition to traditional rehabilitation) or traditional rehabilitation only. Both groups participated in a 4-wk protocol with 3 exercise sessions per day. Before the study, pain intensity, functional ability, functional performance, and balance were assessed using outcome measures. The traditional rehabilitation protocol for both groups contained knee muscle strengthening exercises and flexibility exercises for gastrocnemius, iliotibial band, and hamstring muscles. The intervention group also participated in neuromuscular training of the core where they would contract their deep abdominal muscles to activate the core.

VAS, Anterior Knee Pain Scale, step-down test, and Y-balance test

$1 b$

7/10 on PEDro score

There were significant improvements in pain for those who completed the rehabilitation protocol that incorporated core exercises than those who only completed a traditional rehabilitation protocol. In addition, those in the core exercise program also had improvements in their balance and functional performance compared with the traditional rehabilitation protocol group.

\section{Chevidikunnan et $\mathbf{a l}^{8}$}

RCT

A total of 20 female participants with diagnosed PFP (intervention group: age $=$ $22[1] \mathrm{y}$, height $=158.7[5.1] \mathrm{cm}$, weight $=$ 64.8 [8.4] kg; traditional rehabilitation group: age $=21$ [2] $\mathrm{y}$, height $=160.3$ [4.6] $\mathrm{cm}$, weight $=68.3[7.7] \mathrm{kg}$ ) were used in this study. All subjects must have complained of PFP for $4 \mathrm{wk}$.

The participants were randomized into 2 groups: intervention $(n=10)$ and traditional rehabilitation $(n=10)$. The intervention group completed core muscle strengthening exercises in addition to a traditional rehabilitation protocol 3 times/wk for $4 \mathrm{wk}$. Each session lasted between 30 and $45 \mathrm{~min}$ consisting of a brief warm-up and cooldown. The core exercises consisted of curlups, side bridges, and quadruped positions. The traditional rehabilitation intervention consisted of eccentric quadriceps strengthening and abductor and external rotators strengthening.

VAS and Star Excursion Balance Test $1 b$

\section{6/10 on PEDro score}

The intervention group reported significant improvement in pain based on the VAS. They also displayed better dynamic balance as measured by the Star Excursion Balance Test. ments in resolution of pain. In addition, those in the hip- and core-focused group improved their core endurance.

Abbreviations: PFP, patellofemoral pain; RCT, randomized control trial; VAS, visual analog scale.

the same subject group; thus, 2 were removed. The study by Ferber et al, ${ }^{7}$ Motealleh et al, ${ }^{9}$ and Chevidikunnan et $\mathrm{al}^{8}$ were used for the purpose of this critically appraised topic (CAT) due to pain being the primary outcome explored. The final 3 studies $^{7-9}$ are presented in Table 2.

\section{Best Evidence}

The studies included were identified as the best match in accordance with our predetermined inclusion and exclusion criteria. All studies used pain as at least one of their outcome measures. These studies compared core exercises with traditional rehabilitation for treatment of pain in PFP patients.

\section{Implications for Practice, Education, and Future Research}

The aim of this CAT was to determine if the implementation of core stability exercises coupled with traditional rehabilitation has a greater pain reduction in patients with PFP versus traditional rehabilitation alone. The 3 studies examined in this CAT support the notion that core stability contributes to pain reduction in patients with PFP. Two studies ${ }^{8,9}$ reported a significant reduction in pain at the end of a 4-week core strengthening intervention protocol compared with the traditional rehabilitation group. One study ${ }^{7}$ reported similar reductions in pain between the intervention and traditional rehabilitation groups after a 6-week protocol; 
however, the intervention group reported a significant reduction in pain a week earlier.

The intervention period duration differed across the studies, as did treatment sessions. Chevidikunnan et $\mathrm{al}^{8}$ and Motealleh et al ${ }^{9}$ used a 4-week supervised intervention period, with 3 sessions per day, whereas Ferber et $\mathrm{al}^{7}$ had an intervention period of 6 weeks with 6 sessions per week, 3 sessions per week being under the supervision of a certified athletic trainer. The traditional rehabilitation group in all 3 studies performed open-kinetic and closedkinetic chain exercises to target the quadriceps musculature. The intervention groups had additional assigned exercises to challenge the core musculature. Chevidikunnan et $\mathrm{al}^{8}$ incorporated what was labeled the "big 3" core exercises: cross curl-up, side bridge, and quadruped stance. Motealleh et $\mathrm{al}^{9}$ core exercises included bridging, side-lying hip abduction, lateral straight leg raises, curl-ups, standing isometric hip abduction, single-leg intermittent shoulder flexion/extension, single-leg trunk rotation, and lateral curl-ups. Ferber et $\mathrm{al}^{7}$ instructed patients to stabilize their core musculature before initiation exercises, which included seated hip external rotation, standing hip abduction, standing hip internal rotation, standing hip external rotation, double-leg balance, and single-leg balance on an Airex (Airex AG, Switzerland). Given these differences among the studies and the comparable outcomes regarding reported pain, one can recommend that a 4-week intervention consisting of at least 3 sessions per week targeting core musculature is sufficient to reduce pain in patients with PFP. The rehabilitation protocols used in these studies included exercises that targeted core musculature in 3 planes: sagittal, frontal, and transverse. The protocols also included balance exercises requiring whole-body coordination (ie, quadruped position/bird dog exercises) and addressed strengthening the hip musculature. Similar exercises should be considered in a targeted PFP rehabilitation protocol.

All 3 studies evaluated self-reported pain through a 100-mm VAS. Two studies determined a $20 \mathrm{~mm}$ improvement as clinically meaningful, ${ }^{7,8}$ with the last study defining clinical significance at 10.1 to $30 \mathrm{~mm} .{ }^{9}$ The $20 \mathrm{~mm}(2 \mathrm{~cm})$ has been reported as a valid and reliable minimal clinical important difference for pain in PFP patients. ${ }^{11}$ Self-reported pain was taken at different time points throughout the studies. Chevidikunnan et $\mathrm{al}^{8}$ and Motealleh et $\mathrm{al}^{9}$ only took baseline and postintervention values making it unclear at what point in the 4-week rehabilitation program patients begin to experience clinically meaningful improvements in their pain. Whereas Ferber et $\mathrm{al}^{7}$ collected weekly measurements, making it clear that patients were experiencing clinically meaningful results at week 3 , one week earlier than the traditional rehabilitation group. Health care professionals should consider using a VAS or other patient-reported outcome measures weekly for more accurate tracking of self-reported pain. Additional outcome measures should be used at a clinician's discretion depending on the patient's sport, level of function, activities of daily living, patient goals, and so on.

Traditional rehabilitation, shared by both the core rehabilitation and comparative groups, may have strengthened the knee and hip complex, possibly contributing to favorable results in self-reported pain. Our results agree with previous literature that suggests patients may experience additional benefits from utilizing hip and core strengthening..$^{10}$ Practicing clinicians should incorporate both traditional rehabilitation and core stability rehabilitation for ideal results. Overlapping muscle activation could have influenced the outcome measures for both the core rehabilitation and traditional rehabilitation groups. Future research should aim to determine which corerelated muscles are the best to target to improve self-reported pain in patients with PFP and the optimal frequency and volume of exercise. In addition, the repetition of these studies with a larger sample size may improve the robustness of these results.

Based on the evidence, we suggest that neuromuscular training at the core be considered as an addition to PFP rehabilitation protocols. Further research is needed to determine the long-term benefits of core stability in limiting the recurrence of PFP that is currently lacking. This CAT should be reviewed in 2 years to determine whether additional best-research evidence has been published that could aid in answering the focused clinical question.

\section{References}

1. Bolgla LA, Boling MC, Mace KL, DiStefano MJ, Fithian DC, Powers CM. National athletic trainers' association position statement: management of individuals with patellofemoral pain. J Athl Train. 2018;53(9): 820-836. PubMed ID: 30372640 doi:10.4085/1062-6050-231-15

2. Smith BE, Selfe J, Thacker D, et al. Incidence and prevalence of patellofemoral pain: a systematic review and meta-analysis. PLoS One. 2018;13(1):e0190892. PubMed ID: 29324820 doi:10.1371/ journal.pone.0190892

3. Boling MC, Nguyen AD, Padua DA, Cameron KL, Beutler A, Marshall SW. Gender-specific risk factor profiles for patellofemoral pain. Clin J Sport Med. 2019;31:49-56.

4. Glaviano NR, Baellow A, Saliba S. Physical activity levels in individuals with and without patellofemoral pain. Phys Ther Sport. 2017;27: 12-16. PubMed ID: 28780340 doi:10.1016/j.ptsp.2017.07.002

5. Lack S, Barton C, Sohan O, Crossley K, Morrissey D. Proximal muscle rehabilitation is effective for patellofemoral pain: a systematic review with meta-analysis. Br J Sports Med. 2015;49(21):13651376. PubMed ID: 26175019 doi:10.1136/bjsports-2015-094723

6. Bolgla LA, Earl-Boehm J, Emery C, Hamstra-Wright K, Ferber R. Pain, function, and strength outcomes for males and females with patellofemoral pain who participate in either a hip/core- or knee-based rehabilitation program. Int J Sports Phys Ther. 2016;11(6):926-935. PubMed ID: 27904794

7. Ferber R, Bolgla L, Earl-Boehm JE, Emery C, Hamstra-Wright K. Strengthening of the hip and core versus knee muscles for the treatment of patellofemoral pain: a multicenter randomized controlled trial. J Athl Train. 2015;50(4):366-377. PubMed ID: 25365133 doi:10.4085/1062-6050-49.3.70

8. Chevidikunnan MF, Al Saif A, Gaowgzeh RA, Mamdouh KA. Effectiveness of core muscle strengthening for improving pain and dynamic balance among female patients with patellofemoral pain syndrome. J Phys Ther Sci. 2016;28(5):1518-1523. PubMed ID: 27313363 doi: $10.1589 /$ jpts. 28.1518

9. Motealleh A, Mohamadi M, Moghadam MB, Nejati N, Arjang N, Ebrahimi N. Effects of core neuromuscular training on pain, balance, and functional performance in women with patellofemoral pain syndrome: a clinical trial. J Chiropr Med. 2018;18(1):9-18. doi:10. 1016/j.jcm.2018.07.006

10. Earl-Boehm JE, Bolgla LA, Emory C, Hamstra-Wright KL, Tarima S, Ferber R. Treatment success of hip and core or knee strengthening for patellofemoral pain: development of clinical prediction rules. J Athl Train. 2018;53(6):545-552. PubMed ID: 29893604 doi:10.4085/ 1062-6050-510-16

11. Crossley KM, Bennell KL, Cowan SM, Green S. Analysis of outcome measures for persons with patellofemoral pain: which are reliable and valid? Arch Phys Med Rehabil. 2004;85(5):815-822. PubMed ID: 15129407 doi:10.1016/S0003-9993(03)00613-0 